\title{
Reactivation of intestinal CMV in a renal transplant patient after I 0 years from the transplant
}

\author{
Maria Landi', Walter De Simone', Elio De Nisco', Raffaele Ariola', Franca Romeo', \\ Pia Carmen Melillo', Generoso Violano', Anna Todisco' \\ I Unità Operativa Complessa di Virologia, Dipartimento di Medicina di Laboratorio, AORN “S.G. Moscati”, Avellino \\ 2 Unità Operativa Complessa di Nefrologia e Dialisi, Dipartimento di Nefrologia - Urologico,AORN “S.G. Moscati”,Avellino
}

Key words: CMV reactivation, Renal transplant, 10 years

Riattivazione di CMV a livello intestinale in paziente trapiantato renale a distanza di 10 anni dal trapianto

\section{SUMMARY}

Introduction. We analyzed the clinical case of a $5 \mathrm{I}$ years old man, kidney transplanted on December 2002.

On April 20l I, he had acute rectal bleeding, renal chronic rejection (creatinine $2.9 \mathrm{mg} / \mathrm{dl}$ ), $\mathrm{Hgb} 8.7 \mathrm{~g} / \mathrm{dl}$, positive anti-CMV antibodies ( $(g G)$. A colonoscopy showed diverticulosis of the rectum associated with deepithelialisation. The patient was treated with maintenance immunosuppressive post-transplant therapy.

On June 20I I, the colonoscopy showed a stenosing lesion of the sigmoid colon, and blood sampling and intestinal biopsy were performed to search Cytomegalovirus (CMV) DNA by PCR.

Methods. The presence of CMV-DNA was sought by automatic extractor QIACUBE, using QIAamp DNA BLOOD Mini Kit (Qiagen) for whole blood and QIAamp DNA Mini Kit (Qiagen) for biopsy. The extracted DNA was then amplified by Real Time PCR using Q-CMV RealTime Complete Kit (Nanogen), on instrument Applied Biosystems 7300.

Results. At disease onset the viral load in whole blood was $208000 \mathrm{Geq} / \mathrm{ml}$, and biopsy was positive.

Antiviral therapy with Ganciclovir led to the negativity of the viral load and remission of symptoms.

Conclusions. The clinical case described presented a reactivation of CMV infection in the intestine after more than 10 years from kidney transplantation, while the highest incidence of $\mathrm{CMV}$ reactivation usually occurs during the first year.

In our opinion, the reactivation can be traced to long-term immunosuppressive therapy (maintenance posttransplant therapy) in combination with a state of inflammation of the intestinal mucosa. In fact, patients with IBD treated with steroid drugs, in particular the group of refractory to therapy and thus have a recovery of the inflammatory process, are exposed to reactivation of CMV with intestinal localization.

\section{INTRODUZIONE}

Il 40-80\% degli individui nei paesi industrializzati e la quasi totalità degli individui nei paesi in via di sviluppo vanno incontro ad infezione da Cytomegalovirus umano (HCMV) (Figura I) che, nei soggetti con buone funzioni immunitarie, decorre, di norma, in modo asintomatico. L'infezione da HCMV, al contrario, è un'importan-

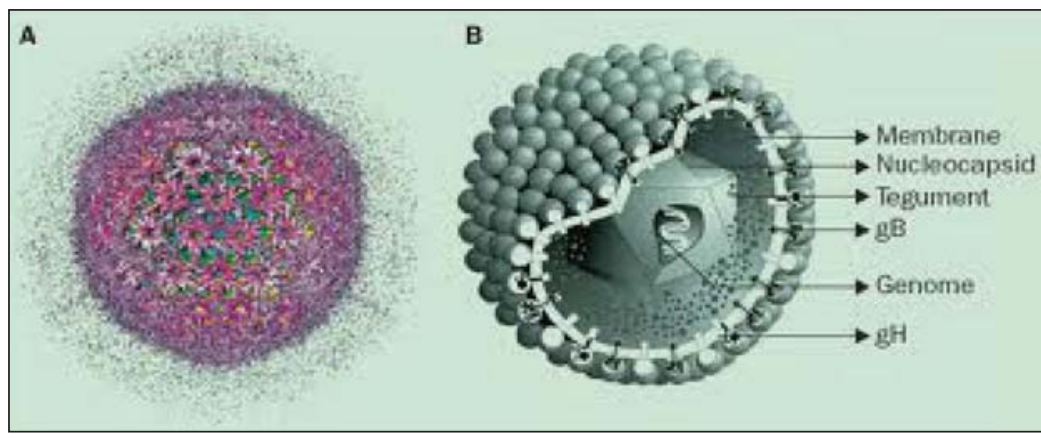

Figura I. Cytomegalovirus. te causa di morbilità e mortalità nei pazienti sottoposti a trapianto di cellule staminali ematopoietiche (HSCTR) e di organo solido (SOD) $(1,2,6)$.

Il caso clinico alla nostra osservazione riguarda un uomo di 51 anni trapiantato di rene nel dicembre del 2002.

Ricoverato nel reparto di Nefrologia e Dialisi nell'aprile 2011 per rettorragia acuta presentava, agli esami di laboratorio, alterazioni della funzionalità renale da rigetto cronico (Creatininemia 2.9 $\mathrm{mg} / \mathrm{dl}$ ) ed alterazioni emocromocitometriche ( $\mathrm{Hgb} 8.7 \mathrm{gr} / \mathrm{dl}$ ). Presentava positività sierologica per anticorpi anti-CMV (IgG). La colonscopia evidenziava diverticolosi del retto con associata disepitelizzazione.

Il paziente era in trattamento con terapia immunosoppressiva di

Corresponding author: Anna Todisco

Tel./Fax 0825203759

E-mail: antodisco@aosgmoscati.av.it 
mantenimento post-trapianto, comprensiva di terapia cortisonica blanda (Urbason $6 \mathrm{mg} / \mathrm{die}$ ).

Ad un secondo ricovero a giugno dello stesso anno, necessario per il persistere e l'aggravarsi della fenomenologia clinica, la colonscopia evidenziava una lesione stenosante del colon sigma (Figura II); venivano effettuati prelievo di sangue e biopsia intestinale per la ricerca di CMV-DNA in PCR.

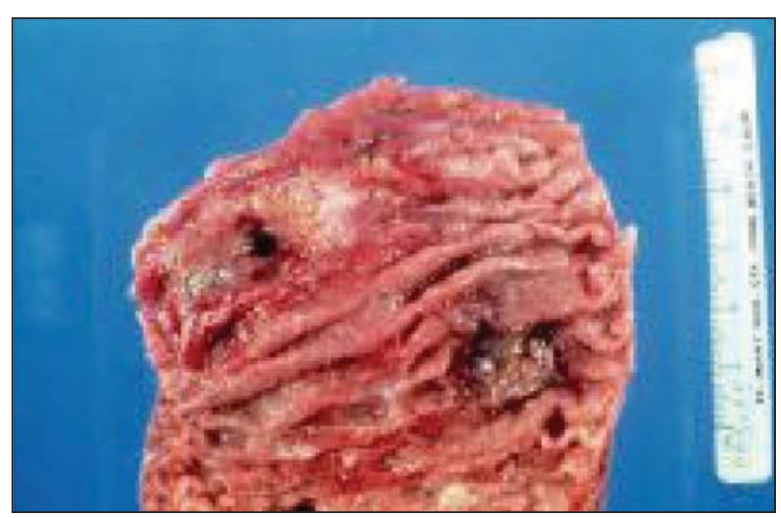

Figura II. Lesione del colon da Cytomegalovirus.

\section{METODI E RISULTATI}

Il CMV-DNA è stato ricercato mediante estrazione automatica di DNA su strumento QIACUBE, utilizzando QIAamp DNA Blood MiniKit (Qiagen, Hilden, Germany) per il sangue intero e QIAamp DNA Mini Kit (Qiagen, Hilden, Germany) per la biopsia; questo sistema semiautomatico ha permesso di evitare contaminazioni e di maneggiare in modo sicuro il campione potenzialmente infetto. Il DNA estratto è stato successivamente amplificato mediante Real Time PCR utilizzando Q-CMV RealTime Complete Kit (Nanogen Advanced Diagnostics, Torino), basato sulle tecnologia Taqman, su strumento Applied Biosystem 7300.

La viral load eseguita su sangue intero all'esordio della malattia era di $208000 \mathrm{gEq} / \mathrm{ml}$, la PCR eseguita su biopsia risultava positiva al virus (ricerca qualitativa). Ad un successivo controllo, dopo terapia antivirale, si osservava la negativizzazione della carica virale su sangue.

\section{CONCLUSIONI}

Il caso clinico descritto presenta la peculiarità di una riattivazione di infezione citomegalica a livello intestinale a distanza di ben 10 anni dal trapianto di rene, laddove la maggiore incidenza di riattivazione da CMV si manifesta generalmente nel corso del primo anno dal trapianto.

La nostra ipotesi è che la riattivazione possa essere ricondotta alla terapia immunosoppressiva di lunga durata a cui il paziente era sottoposto (terapia di mantenimento post-trapianto) in combina- zione con uno stato flogistico della mucosa intestinale.

A conforto di tale ipotesi, numerosi studi segnalano che pazienti con IBD in trattamento con farmaci steroidei, in particolare il gruppo di refrattari alla terapia e che quindi presentano una ripresa del processo infiammatorio, sono esposti a riattivazione dell'infezione citomegalica, con localizzazione intestinale $(3,5)$ (Figura III).
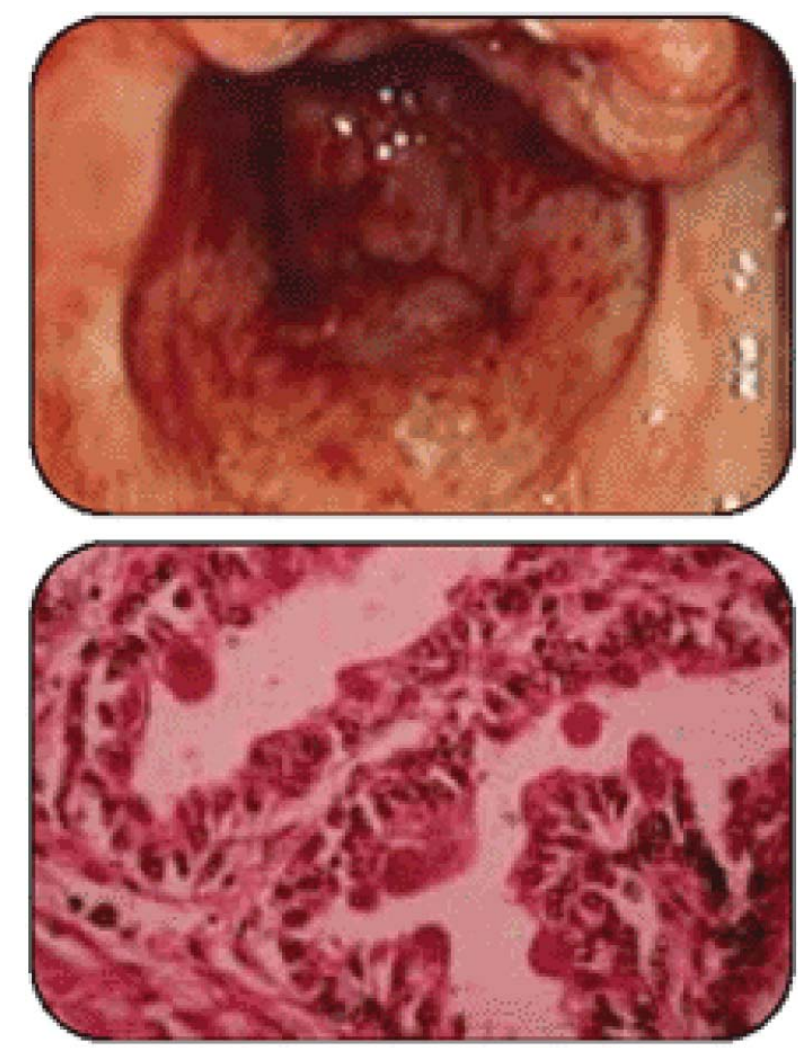

Figura III. Localizzazione intestinale dell'infezione da Cytomegalovirus.

Infatti, sebbene il CMV sia un patogeno noto nelle condizioni di immunodeficienza, negli anni ha assunto una posizione che ha suscitato l'attenzione anche nelle condizioni di infiammazione. L'immunodepressione indotta dalla stessa malattia o dai farmaci steroidei utilizzati per il mantenimento della remissione può influire sulla riattivazione del virus, che manifesta tropismo verso le aree di infiammazione intestinale.

Si ipotizza che il virus, nel momento in cui interagisce con l'ospite, scatena un'attivazione di citochine (IL-6, IL-12, TNF $\alpha$, INF $\gamma$ ) responsabili dell'innesco o del mantenimento dello stato infiammatorio (7).

A conforto del ruolo eziologico svolto dal CMV nell'esacerbazione della malattia, dati di letteratura riportano remissione della fase di riacutizzazione e riduzione della morbilità dopo trattamento dell'infezione con terapia antivirale (Ganciclovir 
o Foscarnet) $(4,8)$.

Analogamente, il nostro paziente, trattato con Ganciclovir per 15 gg., presentava, al termine della terapia, assenza di viremia su sangue e remissione della sintomatologia clinica (dolore addominale e rettorragia).

\section{BIBLIOGRAFIA}

1. Gerna G, Lilleri D, Caldera D, et al. Validation of a DNAemia cutoff for preemptive therapy of cytomegalovirus infection in adult hematopoietic stem cell transplant recipients. Bone Marrow Transplant 2008; 41 : 873-9.

2. Gerna G. Management of human cytomegalovirus infection in Hematopoietic stem cell transplant recipients. Guidelines of the "Società Italiana di Virologia"- SIV.15 giugno 2009.

3. Gilat T, Hacohen P, Lilos P. Childhood factors in ulce- rative colitis and Crohn's disease. Scand $J$ Gastroenterol 1987; 22: 1009-24.

4. Hamlin PJ, Shah MN, Scott N, Wyatt JI, Howdle PD. Systemic cytomegalovirus infection complicating ulcerative colitis; a case report and review of the literature. Postgraduate Medical Journal, 2004; vol 80; 233-5.

5. Mee AS, Jewell DP. Factors inducing relapses in IBD. Br Med J 1978; 2: 6140-801-2.

6. Paya CV. Prevention of cytomegalovirus disease inrecipients of solid-organ transplants. Clin Infect Dis 2001; 32: 596-603.

7. Rahbar A, Bostrom L, Lagerstedt U, et al. Evidence of active cytomegalovirus infection and increased production of IL-6 in tissue specimens obtained from patients with inflammatory bowel diseases. Infl amm Bowel Dis 2003; 9: 154-61.

8. Rajal Khan, et al. Cytomegalovirus colitis followup azacitidine therapy. Can J Infect Dis Med Microbiol 2011; 22 (3). 